\title{
Attachment styles and PTSD in adolescents in three Nordic Countries
}

\author{
Tóra Petersen", Ask Elklit \\ National Centre for Psychotraumatology, University of Southern Denmark, Odense, Denmark \\ Email: ${ }^{*}$ tpetersen@,health.sdu.dk
}

Received 10 February 2013; revised 16 March 2013; accepted 26 March 2013

Copyright (C) 2013 Tóra Petersen, Ask Elklit. This is an open access article distributed under the Creative Commons Attribution License, which permits unrestricted use, distribution, and reproduction in any medium, provided the original work is properly cited.

\section{ABSTRACT}

Studies of possible variables influencing development of posttraumatic stress (PTSD) are of great importance in the effort to enhance preventive and interventional methods in the aim to prevail this devastating condition. Despite the vast research in adults, little is still known of adolescents. Although research is scarce, attachment style has been identified to be an influencing factor in adults, and still research in adolescent is almost non-existent. To our knowledge, no prior study has been conducted, looking at the relationship between attachment style, exposure to a broad range of potentially traumatic events, and subsequent PTSD symptoms in a cross cultural perspective. This is the aim of the present study. Procedure: Data was collected from questionnaire surveys of 1283 adolescents (mean age 14.1 years). Measures: PTSD was measured with The Harvard Trauma QuestionnairePart IV (HTQ); and attachment style was studied using the Revised Adult Attachment Scale (RAAS). Potentially traumatic events were examined using a broad event list of 19 events. Results: Contrary to what was expected, significant cross cultural differences were found in the distribution of securely, preoccupied, and dismissive attached. Around half of the Faroese population was securely attached, contrary to the Danish and Icelandic population, where the majority was dismissive attached. Cross cultural differences were also found in the distribution of attachment style among those adolescents with PTSD. The majority of the Danish adolescent population with PTSD was dismissive attached; the majority of the Faroese adolescent population with PTSD was securely attached, and in the Icelandic adolescents with PTSD were distributed somewhat even between the four attachment styles. Finally, cross cultural differ-

\footnotetext{
${ }^{*}$ Corresponding author.
}

ences were identified in which potentially traumatic events had a significant contribution to the variation in PTSD. The results are discussed and clinical recommendations are presented.

Keywords: Attachment; Posttraumatic Stress Disorder; Traumatic Events; Negative Life Events; Gender; Adolescents

\section{INTRODUCTION}

Since posttraumatic stress disorder (PTSD) was included in the Diagnostic and Statistical Manual-III (DSM) in 1980 [1], the number of studies focusing on adolescents and PTSD has increased. The high number of studies indicates that this population, like adults, is in risk of developing posttraumatic stress symptoms after exposure to traumatic events [2]. This research; however, is mostly based on specific trauma populations [3-7], as for instance clinical or convenient samples [8-10], and studies of broad trauma populations are still sparse.

Existing research shows that not all develop PTSD following exposure to potentially traumatic events. Therefore, studies contributing to a better understanding of the development of PTSD are of great importance. Studies of influencing variables can elucidate underlying causality, and thereby improve prevention and intervention. Some variables which have been identified to predict elevated risk in later psychopathology in children and adolescents are low socioeconomic status and parental psychopathology. And in the attempt to explain this correlation, studies have aimed to identify possible mediating factors. Attachment style is one possible mediating factor that has been found to explain psychopathology in general [11]; and attachment style has been identified to have an impact on the development of PTSD following exposure to a potentially traumatic event $[12,13]$. The coping processes after a trauma seem to be disrupted in individuals 
characterized by anxious or avoidant attachment, and the risk of developing PTSD and other psychological problems therefore are increased [14].

Attachment theory is the joint work of John Bowl and Mary Ainsworth, and was originally developed to describe attachment patterns between caregiver and child. Attachment theory focuses on emotional development seen in a life-time perspective. The theory suggests that children, through interactions with a caregiver, develop internal working models of themselves and of others [1517]. Adult attachment theory has been further developed by others; Bartholomew and Horowitz [18] have developed a two-dimensional four category model of adult attachment, describing the connection between attachment and models of self and others resulting in four patterns of attachment (secure, preoccupied, dismissive, and fearful; cf. also Stein, Jacobs, Ferguson, et al. [19]). Although limited, studies of child-mother attachment have shown that the majority of children could be characterized by a secure attachment pattern [20]. Literature on attachment in adolescents is however sparse, and to our knowledge, no research exists that studies the distribution of the four attachment styles in adolescent populations [21]. However, some studies focusing on specific events, such as terror attacks and community violence, have indicated an association between adolescent attachment style, trauma, and PTSD symptoms [22,23]. One of the studies is based on a group of incarcerated adolescent females showing, that their cognitions of secure attachment were significantly negative associated with rates of direct exposure to community violence [23]. These findings are supported by other studies, showing that having a secure attachment is associated with less reporting of traumatic life events [24,25].

National epidemiological studies of adolescent attachment in relation to trauma and PTSD symptoms, which focus on a broad range of events, are almost non-existent. Elklit [26] examined the relationship between childhood trauma exposure, psychosocial factors, and mental health in a Danish national representative sample of 390 eighth-grade students ( $M=14.5$ years). In this study, the anxious attachment subscale was associated with increased mental health problems, whereas the dependent and close attachment subscales together with self-worth were associated with decreased mental distress [26].

Likewise, O'Connor and Elklit [27] focused on a broad range of events in their study of PTSD and attachment in a sample consisting of young adults in vocational training $(M=29.2$ years). These results indicated that a fearful attachment style was associated with a higher degree of posttraumatic symptoms, whereas having a secure attachment style was associated with a lower degree of traumatization and trauma related symptoms. Studies of attachment styles and PTSD based on older subjects have shown that having an attachment style characterized by a negative picture of the self is a possible risk factor for PTSD [23,27-29]. That is the preoccupied and the fearful attachment style as defined by Bartholomew and Horowitz [18]; whereas having an attachment style characterized by a positive perception of the self may function as a buffer [30]; that is the secure and the dismissive attachment style [18].

Overall, findings from studies of attachment style and PTSD generally indicate that having a secure attachment style is associated with decreased levels of PTSD symptoms, whereas insecure attachment styles are associated with enhanced levels of PTSD symptoms after exposure to traumatic events. To the best of the authors' knowledge, the present research is the first study of the relationship between attachment style, exposure to a broad range of potentially traumatic events, and subsequent PTSD symptoms in a cross cultural perspective.

The first aim of the present study was to explore the distribution of the attachment patterns in the three Nordic countries. The second aim was to examine the distribution in attachment style among those adolescents suffering from PTSD. The final aim was to examine the contribution of gender, attachment style, and 19 potentially traumatic events and negative life events to the variance in PTSD.

\section{METHOD}

\subsection{Subjects}

The data was collected from questionnaire surveys of adolescents from two national representative probability samples from Denmark ( $n=390$, mean age 14.5 years), Iceland ( $n=206$, mean age 14.5 years), and from a total population sample from the Faroe Islands $(n=687$, mean age 14.2 years). The total sample was 1283 adolescents with a mean age of 14.1 years $(S D=1.57) .48 \%(n=615)$ of the total sample were males and $51 \%(n=648)$ were females. Twenty adolescents $(1.6 \%)$ did not report their gender.

\subsection{Procedure}

The questionnaires were distributed in primary public schools in the three Nordic countries. The headmaster of the schools received a letter, where they were invited to participate. In the Faroe Islands all schools teaching eighth grade students received a letter. All schools but one accepted to participate; however only one eighth grade student was in the school not participating [10]. In Denmark [26] and Iceland [8] the schools were collected from a list of all schools in the country. The samples were geographically stratified with sample allocation proportionate to the population distribution. The schools 
invited were chosen by permutation. Primary public schools are practically obligatory in the three countries; therefore students in those schools constitute a good representation of the adolescent population.

\subsection{Measures}

The questionnaire included a list of 19 potentially traumatic events and negative life events. The students were asked to indicate for each event, whether they had been exposed to it. The events were selected from relevant literature and clinical experience, and were as follows: traffic accident, other serious accidents, physical assault, rape, witnessed other people injured or killed, came close to being injured or killed, threatened to be beaten, neardrowning, robbery/theft, pregnancy/abortion, serious illness, death of someone close, divorce of parents, sexual abuse, severe childhood neglect, humiliation or persecution by others (bullying), and absence of a parent.

The Harvard Trauma Questionnaire-Part IV (HTQ) [31] consists of 31 items and is used to estimate the occurrence of PTSD at the time of the event chosen as the most distressing event by the adolescent. The items were scored on a 4-point Likert scale ( $1=$ not at all present; 4 = present extremely often). The HTQ-Part IV permits an assessment of whether or not a person suffers from PTSD. The HTQ-Part IV measures the intensity of the three core symptom groups of PTSD: Intrusion, Avoidance, and Arousal. Only symptoms scored "quite a bit" or "extremely" counted for a PTSD diagnosis. The subscales were scored separately. The original Mollica et al. article [31] found good reliability and validity for the scale. The internal reliability of the scale in the present populations was high, as the Cronbach's $\alpha$ was 0.96 for the PTSD scale.

The revised Adult Attachment Scale (RAAS) [32,33] is an 18-item self-report scale based on attachment theories $[16,18]$. The items are scored on a 5-point Likert scale $(1=$ not at all characteristic; $5=$ very characteristic of me). The closeness and dependency subscales are combined, and two subscales are used in establishing four attachment patterns. The close-dependency subscale consists of 12 items. The internal reliability in the sample was low as the Cronbach's $\alpha$ was 0.42 . Despite the lim- ited reliability, it was decided to continue to use this subscale for constructing attachment patterns. The Anxiety subscale consists of six items, and showed an acceptable internal reliability (Cronbach's $\alpha=0.75$ ). The four attachment patterns are: secure attachment (closedependency score above 36, and an anxiety score under 18), preoccupied attachment (close-dependency scores above 36 and an anxiety score above 18, dismissive attachment (close-dependency score under 36, and an anxiety score under 18, and finally the fearful attachment (close-dependency score under 36, and an anxiety score above 18).

\subsection{Statistical Analyses}

Pearson correlation analyses were conducted of the four attachment styles separately for each country. In order to examine each variables contribution to the variation in PTSD linear regression analyses were conducted using the HTQ total score as dependent variable. Gender, the four attachment styles, and the 19 potentially traumatic events were imputed as independent variables. Only the events that came out significant after controlling for each variable are reported. The analyses were conducted in SPSS 19.0.

\section{RESULTS}

The cross cultural difference in the distribution of the attachment styles is presented in Table 1. The Pearson Chi-Square was significant for three of the attachment styles: the secure attachment style $\left(\chi^{2}=174.32, \mathrm{p}<\right.$ $0.0005)$; the preoccupied attachment style $\left(\chi^{2}=11.28, \mathrm{p}\right.$ $<0.005)$, and the dismissive attachment style $\left(\chi^{2}=\right.$ $256.31, \mathrm{p}<0.0005)$, but not the fearful attachment style.

Only those adolescents who had filled out all the questions the PTSD-measure (HTQ) and the attachment stylemeasure (RAAS) were included in further analyses (reported in second column in Table 2). The distribution of attachment styles among those adolescents with PTSD was analysed using Pearson Chi Square analysis. The results are presented in Table 2. In the Danish sample, the majority of the adolescents with PTSD were characterized by a preoccupied attachment style. In the

Table 1. The distribution of the four attachment styles in the three countries.

\begin{tabular}{|c|c|c|c|c|}
\hline & $\begin{array}{c}\text { Secure attachment } \\
(N / \% \text { in within the country) }\end{array}$ & $\begin{array}{l}\text { Preoccupied attachment } \\
\text { (N/\% in within the country) }\end{array}$ & $\begin{array}{c}\text { Dismissive attachment } \\
\text { ( } N / \% \text { in within the country) }\end{array}$ & $\begin{array}{c}\text { Fearful attachment } \\
(N / \% \text { in within the country) }\end{array}$ \\
\hline $\begin{array}{c}\text { Denmark }(N=87 \% ; 52 \text { could } \\
\text { not be categorized })\end{array}$ & $14 \%(N=53)$ & $13 \%(N=50)$ & $49 \%(N=191)$ & $11 \%(N=44)$ \\
\hline $\begin{array}{c}\text { Iceland ( } N=87 \% ; 27 \text { could } \\
\text { not be categorized) }\end{array}$ & $12 \%(N=24)$ & $11 \%(N=23)$ & $52 \%(N=107)$ & $12 \%(N=25)$ \\
\hline $\begin{array}{c}\text { Faroe Islands ( } N=87 \% ; 84 \\
\text { could not be categorized) }\end{array}$ & $52 \%(N=358)$ & $18 \%(N=125)$ & $7 \%(N=49)$ & $10 \%(N=71)$ \\
\hline
\end{tabular}

*The remaining \% could not be categorized by one distinct attachment style, and were therefore excluded from the analyses. 
Table 2. The distribution of attachment styles among the adolescents with PTSD.

\begin{tabular}{ccccccc}
\hline \multirow{2}{*}{ Country } & New $N(\%$ of total $N)$ & $\begin{array}{c}\text { Fulfilling the } \\
\text { PTSD criteria }\end{array}$ & & & & \\
& & & & & & \\
Denmark & $357(92 \%)$ & 30 & $6.7 \%$ & $26.7 \%$ & $43.3 \%$ & $16.7 \%$ \\
Iceland & $182(88 \%)$ & 33 & $15.2 \%$ & $27.3 \%$ & $24.2 \%$ & $18.2 \%$ \\
Faroe Islands & $618(90 \%)$ & 132 & $34.8 \%$ & $8.3 \%$ & $25.8 \%$ & $21.2 \%$ \\
\hline
\end{tabular}

Icelandic sample, the adolescents with PTSD were somewhat even distributed across the various attachment styles. In the Faroese sample, the majority of the adolescents with PTSD were characterized by a secure attachment style (Table 2).

Each variable's contribution to the variation in PTSD after controlling for the others variables was examined conducting linear regression analyses for each country. The HTQ total score was used as dependent variable and the results are presented in Tables 3(a)-(c). Also here cross cultural variance was found.

Female gender did not come out significant in the Icelandic sample, but in the other two samples. Having a preoccupied attachment style came out significant as a risk factors for PTSD in the Danish sample, but not the other samples. Dismissive attachment had a buffering effect in the Danish sample, but was none significant in the two other samples, and finally fearful attachment was a significant risk factor in the Faroese sample, but not the other two samples. Moreover exposure to humiliation or bullying was the only event that came out significant in all three samples, the other events varied across the countries (Tables 3(a)-(c)).

\section{DISCUSSION}

The first aim of the present study was to explore the distribution of the attachment styles in the three Nordic countries. Based on previous research in attachment styles in children, we expected to find a majority of securely attached in all three countries. Contrary our expectations we found significant cross cultural differences in the distribution of securely attached, preoccupied, and dismissive attached. As expected the majority of the Faroese adolescent sample was securely attached, contrary the majority in the Danish and Icelandic samples was dismissive attached (Table 1). Further there were significantly more Faroese adolescents characterized by a preoccupied attachment style, compared to the other two countries.

To our knowledge epidemiological studies on attachment style among adolescents are non-existent, which hinders a direct comparison. However a study based on a convenience US sample of older high school students ( $M$ $=16.6$ years) has shown that $59 \%$ of the participants were securely attached and only eight per cent were
Table 3. (a) Danish Sample. Model summary: R Square $=0.40$, $\mathrm{F}=6.74, \mathrm{p}<0.0005$; (b) Icelandic Sample. Model summary: $\mathrm{R}$ Square $=42, F=3.40, p<0.0005$; (c) Faroese Sample. Model summary: $R$ Square $=0.40, F=13.96, p<0.0005$.

(a)

\begin{tabular}{ccc}
\hline Independent Variable & $\mathrm{t}$ & $\mathrm{p}$-value \\
\hline Female gender & 3.74 & 0.0005 \\
Secure attachment & -0.61 & $\mathrm{~ns}$ \\
Preoccupied attachment & 3.52 & 0.001 \\
Dismissive attachment & -1.98 & 0.05 \\
Fearful attachment & 1.67 & $\mathrm{~ns}$ \\
18. Exposure to humiliation or bullying & 3.05 & 0.005 \\
\hline
\end{tabular}

(b)

\begin{tabular}{ccc}
\hline Independent Variable & $\mathrm{t}$ & $\mathrm{p}$-value \\
\hline Female Gender & -1.13 & $\mathrm{~ns}$ \\
Secure attachment & 0.10 & $\mathrm{~ns}$ \\
Preoccupied attachment & 0.42 & $\mathrm{~ns}$ \\
Dismissive attachment & -2.19 & 0.03 \\
Fearful attachment & 0.13 & $\mathrm{~ns}$ \\
4. Exposure to rape & 2.78 & 0.01 \\
12. Exposure to serious illness & -2.04 & 0.05 \\
18. Exposure to humiliation or bullying & 1.99 & 0.05 \\
\hline
\end{tabular}

(c)

\begin{tabular}{ccc}
\hline Independent Variable & $\mathrm{t}$ & $\mathrm{p}$-value \\
\hline Female gender & 8.48 & 0.0005 \\
Secure attachment & -0.93 & $\mathrm{~ns}$ \\
Preoccupied attachment & 0.79 & $\mathrm{~ns}$ \\
Dismissive attachment & 0.27 & $\mathrm{~ns}$ \\
Fearful attachment & 3.34 & 0.001 \\
2. Exposure to serious accident & 4.0 & 0.0005 \\
9. Attempted suicide & 3.71 & 0.0005 \\
11. Having been pregnant/abortion & -2.31 & 0.02 \\
18. Exposure to humiliation or bullying & 4.43 & 0.0005 \\
19. Absence of a parent & 2.96 & 0.005 \\
\hline
\end{tabular}

characterized by a preoccupied attachment style [29]. Another study of young adults $(M=19.6$ years) found $14 \%$ to be characterized by a preoccupied attachment style and $18 \%$ by a dismissive attachment style. In this study almost twice as many $(21 \%)$, as in the present study $(10 \%-12 \%)$, were fearfully attached [18]. 
Besides the differences in the samples used in the mentioned studies, a possible explanation of the high occurrence of dismissive attached in the Danish and the Icelandic samples may be that the present study is based on younger adolescent samples, and though it is theorized that attachment style is a stable factor through life [34], adolescents might be characterized by more unstable attachment styles [17]. Adolescence is a period characterized by hormonal, physical, and social changes. The adolescents search for a new identity in order to match the changes, and are characterized by increased detachment from the parents [35], and feelings of distress and insecurity may be prominent [36]. The major cross cultural difference in the occurrence of dismissive attached is interesting and could be explained by the variations in the cultures [37]. The Faroese society is still more collectivistic oriented compared to the Danish and Icelandic societies, which could explain, that Faroese adolescents initiate the detachment process from their parents at an older age, compared to adolescents in more individualistic oriented cultures. To examine this, future research ought to replicate the present study in the same countries but in older adolescents or young adults.

The second aim of the study was to examine the distribution in attachment style among those adolescents suffering from PTSD. It was expected that adolescents suffering from PTSD either were characterized by a preoccupied or a fearful attachment style.

In the Danish and the Icelandic sample we partly found what was expected, as the fewest of the adolescents with PTSD were characterized by a secure attachment style. This finding is similar to a study comparing adolescents with learning disabilities and a non-clinical control group, which found that anxious attachment style, was a risk factor of PTSD [22]. In the Danish and Icelandic samples however, more than one fourth of the adolescents with PTSD had a dismissive attachment style, and only one sixth had a fearful attachment style. Contrary this, other studies have found that a preoccupied, dismissive or fearful attachment style generally is associated with higher levels of psychopathology [38].

The results from the Faroese sample were somewhat different. The majority of the adolescents suffering from PTSD were securely attached, which is contrary other studies that have found that the securely attached are flexible in their coping and have most success in maintaining good adjustment [39]. In the Faroese sample the smallest percentage of those with PTSD was dismissive attached, one fourth was preoccupied and one fifth was fearfully attached. In comparison, a study of adults exposed to the September 11 attack showed that securely attached had fewer symptoms of PTSD compared to those with the three other attachment styles [40]. This study also examined the participants' psychological ad- aptation by asking their relatives and friends. Also here the securely attached were described by the best adaptation, and in concordance with the present study, those participants with preoccupied attachment style, were described as least psychologically adapted after the attack. The dismissive participants in the September 11 attack study described themselves as distressed, and were described by their relatives as less psychological adapted than the secure participants seven months after the attack. However, 18 months after the attack, this difference disappeared. The fearfully attached had high self-reported distress, but were described as above average in psychological adaptation by their relatives [40].

The further linear regression analyses presented in Tables 3(a)-(c) showed that the inserted variables (gender, attachment style, and traumatic events) could explain about $40 \%$ of the variance in PTSD in all three countries; however some interesting cross cultural differences appeared. Female gender contributed strongly to the variance in the Danish and the Faroese samples, although significantly stronger in the Faroese sample. This is in line with research in PTSD, which has found, that the higher risk of PTSD among females not could be explained by trauma type, nor measurement used [41]. This is, however, contrary to the results in the Icelandic sample, where female gender had a negative, but non-significant contribution to PTSD. The present study does not hold information to explain the unexpected results in the Icelandic sample, but it can be assumed, that the explanation could be found in a possible difference in gender roles in the Icelandic society compared to the two other societies [42]. The analysis also showed cross cultural difference in which attachment styles had a significant contribution to PTSD. Other studies have found the dismissive attachment style, characterized by a positive picture of the self and a negative picture of others, to be a protective factor against distress $[18,30]$. This is in concordance to the present results based on the Danish and the Icelandic samples, but not in the Faroese sample. On the other hand and in line with other studies [26] fearful attachment was a significant risk factor in the Faroese adolescents, but not in the Danish and the Icelandic. The secure attachment style did not seem to have a significant contribution to PTSD in the adolescents from any of the three countries. These results therefore are contrary other findings of older populations. A study in undergraduate students (mean age 27 years) found that that low selfesteem was a mediator between childhood trauma and psychopathology in adulthood elevating the risk of psychopathology in adulthood [43]. Another study of trainee firefighters $(M=30$ years $)$ also underlines the importance of view of self or self-worth. Participants' pre- and post-traumatic appraisals were assessed, and a main finding was that maladaptive self-appraisals contributed to 
PTSD [44].

Finally, the cross cultural difference in which of the 19 potentially traumatic events and negative life events contributed significantly to the variance in PTSD controlling for gender and attachment style is noteworthy. Contrary to prior research [5], none of the childhood traumas (sexual abuse, physical abuse, and neglect) came out significant in the regression analyses. However humiliation and bullying had strong significant contribution in all three samples. This might be due to the construction of the questionnaire, as the adolescents can be assumed to choose the most present trauma (bullying) as the most distressing trauma, instead of the childhood trauma. One possible explanation might be that the prior exposure to childhood trauma makes the adolescent more vulnerable to exposure to bullying and more vulnerable to following PTSD [45]. Nevertheless the results underline the importance of using broad event lists when screening for trauma in clinical settings. Two potentially traumatic events: having been pregnant or having had an abortion (in the Faroese sample) and exposure to serious illness (in the Icelandic sample) was found to have a significant negative contribution to PTSD. Although other studies [46] have found those events to induce PTSD, one can also assume that adolescents after exposure to those potentially traumatic events receive great social support and thereby manage to cope with the event in an adaptive way.

Though the retrospective construction of the present study does not allow us to draw any conclusions on causality, and possible mediating factors might explain the findings, Sroufe [47], however, has emphasizes that attachment is placed in the initiation of the developmental processes, through which these potential mediating variables develop. Sroufe [47] found attachment style to be a central variable in a 30-year longitudinal study. He found a lower prevalence of psychopathology among the securely attached in late adolescence and that attachment style significantly associated with many critical developmental functions. The majority of studies on adolescents and trauma have focused on specific, discrete traumatic events. However, studies based on a single type of trauma might fail to see a more comprehensive picture of multiple distressing events, and the impact on the development in adolescence [48]. Additionally, this might lead to ineffective clinical interventions for this age group. Thus, as part of the assessment and planning of interventions, mental health professionals are recommended to integrate a standard procedure to obtain information about stressful events from adolescents. Furthermore, the present study suggests that after exposure to trauma, therapists/ mental health professionals should assess and take into consideration other personal factors such as attachment style $[26,29,49]$. The present results suggest that more research ought to be done in attachment style and PTSD using prospective design.

\section{LIMITATIONS}

The study has a number of limitations. It is based on students' self-reports, which could have produced a response bias, for instance, regarding the students' ability to be factual, their honesty, and their willingness to remember painful events. However, it is likely that the use of the event list was advantageous compared to an interview, because it promotes recognition rather than recall, which is less distressful in the report of emotionally stressful events [50]. Furthermore, the fact that the subjects were adolescents might have produced less of a memory bias, as some events were more recent, compared to similar studies of adult subjects. Moreover, the anonymity of the classroom could have made reporting easier for some subjects, compared to e.g. an interview study. The construction of the questionnaire placed the list of events almost at the end to reduce a biased attitude toward the trauma issue, despite the purpose being mentioned in the introduction. The event questionnaire seems to function well across Western cultures, although it has not been thoroughly validated [26]. Due to the design of the study, there was no way of reporting whether an event had occurred more than once, and hence a distinction between the effect of a single event trauma and repetitive traumas could not have been made.

The low reliability in the attachment measure is an important limitation, and future research ought to replicate the study using validated measurements of adolescent attachment. Moreover, the existence of confounders (e.g. negative affectivity influencing self-reported traumas) is a possibility that might impact the relationships found between attachment styles, events, and PTSD. Despite the limitations, the present paper has several strengths: it is based on representative sample studies from different countries using the same measures, and it reveals important correlations between exposure to potentially traumatic events, PTSD and attachment. Also it identifies cross cultural variation relevant to prevention, intervention, and future research.

\section{REFERENCES}

[1] American Psychiatric Association (1980) Diagnostic and statistical manual of mental disorders. 3rd Edition, American Psychiatric Association, Washington DC.

[2] Caffo, E., Forresi, B. and Lievers, L.S. (2005) Impact, psychological sequelae and management of trauma affecting children and adolescents. Current Opinion in Psychiatry, 18, 422-428. doi:10.1097/01.yco.0000172062.01520.ac

[3] Boney-McCoy, S. and Finkelhor, D. (1995) Psychosocial 
sequelae of violent victimization in a national youth sample. Journal of Consulting and Clinical Psychology, 63, 726-736. doi:10.1037/0022-006X.63.5.726

[4] Broberg, A.G., Dyregrov, A. and Lilled, L. (2005) The Göteborg discotheque fire: Posttraumatic stress and school adjustment as reported by the primary victims 18 months later. Journal of Child Psychology and Psychiatry, 46, 1279-1286. doi:10.1111/j.1469-7610.2005.01439.x

[5] Edgardh, K. and Ormstad, K. (2000) Prevalence and characteristics of sexual abuse in a national sample of Swedish seventeen-year-old boys and girls. Acta Paediatrica, 88, 310-319. doi:10.1111/j.1651-2227.2000.tb01333.x

[6] Evans, L.G. and Oehler-Stinnett, J. (2006) Structure and prevalence of PTSD symptomology in children who have experienced a severe tornado. Psychology in the Schools, 43, 283-295. doi:10.1002/pits.20150

[7] Khamis, V. (2005) Post-traumatic stress disorder among school age Palestinian children. Child Abuse \& Neglect, 29, 81-95. doi:10.1016/i.chiabu.2004.06.013

[8] Bödvarsdóttir, Í. and Elklit, A. (2007) Victimization and PTSD-like states in an Icelandic youth probability sample. BMC Psychiatry, 7, 51.

[9] Elklit, A. and Petersen, T. (2008) Exposure to traumatic events among adolescents in fournations. Torture, 18, 211.

[10] Petersen, T., Elklit, A. and Olesen, J.G. (2010) Victimization and PTSD in a Faroese youth total-population sample. Scandinavian Journal of Psychology, 51, 56-62. doi:10.1111/j.1467-9450.2009.00728.x

[11] Lyons-Ruth, K. and Jacobvitz, D. (2008) Attachment disorganization: Genetic factors, parenting contexts, and developmental transformations from infarncy to adulthood. In: Cassidy, J. and Shaver, P.R., Eds., Handbook of Attachment. Theory, Research, and Clinical Applications. Guilford Press, New York, 666-697.

[12] Declercq, F. and Palmans, J. (2006) Two subjective factors as moderatorsnetween critical incidents and the occurrence of posttraumatic stress disorders: "Adult attachment" and "perception of social support". Psychology and Psychotherapy: Theory, Research and Practice, 79, 232-337. doi:10.1348/147608305X53684

[13] Sandberg, D.A., Suess, E.A. and Heaton, J.L. (2010) Attachment anxiety as a mediator of the relationship between interpersonal trauma and posttraumatic symptomatology among college women. Journal of Interpersonal Violence, 25, 33-49. doi: $10.1177 / 0886260508329126$

[14] Solomon, Z., Dekel, R. and Mikulincer, M. (2008) Complex trauma of war captivity: A prospective study of attachment and post-traumatic stress disorder. Psychological Medicine, 38, 15-28. doi:10.1017/S0033291708002808

[15] Ainsworth, M.S., Blehar, M.C., Waters, E. and Wall, S. (1978) Patterns of attachment: A psychological study of the strange situation. Lawrence Erlbaum, Oxford.

[16] Bowlby, J. (1988) A secure base. Clinical applications of attachment theory. Routledge, London.
[17] Bretherton, I. (1991) The roots and growing points of attachment theory. In: Parkes, C.M., Stevenson-Hinde, J. and Marris, P., Eds., Attachment across the Life Cycle, Tavistock/Routledge, New York, 9-32.

[18] Bartholomew, K. and Horowitz, L.M. (1991) Attachment styles among young adults: A test of a four-category model. Journal of Personality and Social Psychology, 61, 226-244. doi:10.1037/0022-3514.61.2.226

[19] Stein, H., Jacobs, N.J., Ferguson, K.S., Allen, J.G. and Fonagy, P. (1998) What do adult attachment scales measure? Bulletin of the Menninger Clinic, 62, 33-82.

[20] Van Ijzendoorn, M.H. and Sagi-Schwartz, A. (2008) Cross-cultural patterns of attachment: Universal and contextual dimentions. In: Cassidy, J. and Shaver, P.R., Eds., Handbook of Attachment. Theory, Research, and Clinical Applications. Guilford Press, New York, 880-905.

[21] Freidlmeier, W. and Granqvist, P. (2006) Attachment transfer among Swedish and German adolescents: A prospective longitudinal study. Personal Relationships, 13, 261-279. doi:10.1111/j.1475-6811.2006.00117.x

[22] Finzi-Dottan, R., Dekel, R., Lavi, T. and Su'ali, T. (2006) Posttraumatic stress disorder reactions among children with learning disabilities exposed to terror attacks. ComprehensivePsychiatry, 47, 144-151. doi:10.1016/j.comppsych.2005.05.001

[23] Lindberg, N.M. (1998) Attachment cognitions as moderators of posttraumatic stress disorder symptomatology in adolescents exposed to community violence. Dissertation Abstracts International: Section B: The Sciences and Engineering, 58, 6815.

[24] Campini, C. (2006) Attachment style stability, life events, and adjustment across adolescence: A longitudinal study. Dissertation Abstracts International: Section B: The Sciences and Engineering, 66, 6310.

[25] Tobey, C.P. (2001) Parental attachment, adolescent sexual behaviour, and stressful life events. Dissertation $A b$ stracts International: Section B: The Sciences and Engineering, 62, 1602.

[26] Elklit, A. (2002) Victimization and PTSD in a Danish national youth probability sample. Journal of the American Academy of Child \& Adolescent Psychiatry, 41, 174 181. doi:10.1097/00004583-200202000-00011

[27] O'Connor, M. and Elklit, A. (2008) Attachment styles, traumatic events and PTSD: A cross-sectional investigation of adult attachment and trauma. Attachment and $\mathrm{Hu}$ man Development, 10, 59-71. doi:10.1080/14616730701868597

[28] Dieperink, M., Leskela, J., Thuras, P. and Engdahl, B. (2001) Attachment style classification and posttraumatic stress disorder in former prisoners of war. American Journal of Orthopsychiatry, 71, 374-378. doi:10.1037/0002-9432.71.3.374

[29] Howard, M.S. and Medway, F.J. (2004) Adolescents' attachment and coping with stress. Psychology in the Schools, 41, 391-402. doi:10.1002/pits. 10167

[30] Dekel, R., Solomon, Z., Ginzburg, K. and Neria, Y. (2004) Long-term adjustment among Israeli war veterans: The role of attachment style. Anxiety, Stress \& Coping: An 
International Journal, 17, 141-152. doi:10.1080/10615800410001721184

[31] Mollica, R.F., Caspi-Yavin, Y., Bollini, P., Truong, T., Tor, S. and Lavelle, J. (1992) The Harvard trauma questionnaire: Validating a cross-cultural instrument for measuring torture, trauma, and posttraumatic stress disorder in indochinese refugees. Journal of Nervous and Mental Disease, 180, 111-116. doi:10.1097/00005053-199202000-00008

[32] Collins, N.L. and Read, S.J. (1990) Adult attachment, working models, and relationship quality in dating couples. Journal of Personality and Social Psychology, 58, 644-663. doi:10.1037/0022-3514.58.4.644

[33] Collins, N.L. (1996) Working models of attachment: Implications for explanation, emotion, and behaviour. Journal of Personality and Social Psychology, 71, 810-832. doi:10.1037/0022-3514.71.4.810

[34] Hamilton, C.E. (2000) Continuity and discontinuity of attachment from infancy through adolescence. Child Development, 71, 690-694. doi:10.1111/1467-8624.00177

[35] Jackson, S. and Goossens, L. (2008) Handbook of adolescent development. Psychology Press, East Sussex.

[36] Erikson, E.H. (1968) Identity, youth and crisis. W. W. Norton, New York.

[37] Van IJzendoorn, M.H. and Kroonenberg, P.M. (1988) Cross-cultural patterns of attachment: A meta-analysis of the strange situation. Child Development, 59, 147-156. doi:10.1111/j.1467-8624.1988.tb03202.x

[38] Roche, D.N., Runtz, M.G. and Hunter, M.A. (1999) Adult attachment: A mediator between child sexual abuse and later psychological adjustment. Journal of Interpersonal Violence, 14, 184-207. doi: $10.1177 / 088626099014002006$

[39] Mikulincer, M. and Shaver, P.R. (2007) Attachment in adulthood: Structure, dynamics, and change. Guildford Press, New York.

[40] Fraley, R.C., Fazzari, D.A., Bonanno, G.A. and Dekel, S. (2006) Attachment and psychological adaptation in high exposure survivors of the September 11th attack on the World Trade Center. Personality and Social Psychology Bulletin, 32, 538-551. doi: $10.1177 / 0146167205282741$
[41] Tolin, D.F. and Foa, E.B. (2006) Sex differences in trauma and posttraumatic stress disorder: A quantitative review of 25 years of research. Psychological Bulletin, 132, 959-992. doi:10.1037/0033-2909.132.6.959

[42] Ditlevsen, D.N. and Elklit, A. (2010) The combined effect of gender and age on posttraumatic stress disorder: Do men and women show differences in the lifespan distribution of the disorder? Annals of General Psychiatry, $\mathbf{9}$ 32.

[43] Finzi-Dottan, R. and Karu, T. (2006b) From emotional abuse in childhood to psychopathology in adulthood-A path mediated by immature defense mechanisms and selfesteem. The Journal of Nervous and Mental Disease, 194, 616-621. doi:10.1097/01.nmd.0000230654.49933.23

[44] Bryant, R.A. and Guthrie, R.M. (2005) Maladaptive appraisals as a risk factor for posttraumatic stress-A study of trainee firefighters. American Psychological Society, 16, 749-752.

[45] Petersen, T., Armour, C. and Elklit, A. (Submitted) Modelling patterns of negative life experiences and mental health in Faroese adolescents.

[46] Costello, E.J., Erkanli, A., Fairbank, J.A. and Angold, A. (2002) The prevalence of potentially traumatic events in childhood andadolescence. Journal of Traumatic Stress, 15, 99-112. doi:10.1023/A:1014851823163

[47] Sroufe, L.A. (2005) Attachment and development: A prospective, longitudinal study from birth to adulthood. Attachment \& Human Development, 7, 349-367. doi:10.1080/14616730500365928

[48] Shevlin, M. and Elklit, A. (2008) A latent class analysis of adolescent adverse life events based on a Danish national youth probability sample. Nordic Journal of Psychiatry, 60, 218-224. doi:10.1080/08039480801983992

[49] de Zulueta, F. (2006) The treatment of psychological trauma from the perspective of attachment research. Journal of Family Therapy, 28, 334-351. doi:10.1111/j.1467-6427.2006.00356.x

[50] Willis, G.B. and Gonzalez, A. (1998) Methodological issues in the use of survey questionnaires to assess the health effects of torture. Journal of Nervous and Mental Disease, 186, 283-289. doi:10.1097/00005053-199805000-00004 